\title{
Erratum: Comprehensive analysis of photonic-crystal surface-emitting lasers via time-dependent three-dimensional coupled-wave theory [Phys. Rev. B 99, 035308 (2019)]
}

Takuya Inoue, Ryohei Morita, Masahiro Yoshida, Menaka De Zoysa, Yoshinori Tanaka, and Susumu Noda

(Received 8 April 2019; published 17 April 2019)

DOI: 10.1103/PhysRevB.99.169904

In Eqs. (8) and (A6)-(A9) in our original paper, an imaginary unit " $i$ " in front of the wave-coupling term [the fourth term on the right-hand side of Eq. (8) and the last term on the right-hand side of Eqs. (A6)-(A9)] was missing. The correct expression for these equations are as follows:

$$
\begin{aligned}
& \frac{\partial}{\partial t}\left(\begin{array}{c}
R_{x} \\
S_{x} \\
R_{y} \\
S_{y}
\end{array}\right)=\frac{c}{n_{g}}\left[-i \delta+\frac{g-\alpha_{i n}}{2}\right]\left(\begin{array}{c}
R_{x} \\
S_{x} \\
R_{y} \\
S_{y}
\end{array}\right)-\frac{c}{n_{g}}\left(\begin{array}{c}
\partial R_{x} / \partial x \\
-\partial S_{x} / \partial x \\
\partial R_{y} / \partial y \\
-\partial S_{y} / \partial y
\end{array}\right)-\gamma\left(\begin{array}{c}
R_{x} \\
S_{x} \\
R_{y} \\
S_{y}
\end{array}\right)+\frac{i c}{n_{g}} \mathbf{C}\left(\begin{array}{c}
R_{x} \\
S_{x} \\
R_{y} \\
S_{y}
\end{array}\right)+\left(\begin{array}{l}
f_{1} \\
f_{2} \\
f_{3} \\
f_{4}
\end{array}\right), \\
& \frac{\partial R_{x}}{\partial t}=\frac{c}{n_{g}}\left(-i \delta+\frac{g}{2}\right) R_{x}-\frac{c}{n_{g}} \frac{\partial R_{x}}{\partial x}-\gamma R_{x}+\frac{i c}{n_{g}} \cdot \frac{-\omega_{0}^{2}}{2 \beta_{0} c^{2}} \sum_{m^{\prime}, n^{\prime}} \int \xi_{1-m^{\prime},-n^{\prime}} E_{y, m^{\prime}, n^{\prime}} \Theta_{0}^{*}(z) d z, \\
& \frac{\partial S_{x}}{\partial t}=\frac{c}{n_{g}}\left(-i \delta+\frac{g}{2}\right) S_{x}+\frac{c}{n_{g}} \frac{\partial S_{x}}{\partial x}-\gamma S_{x}+\frac{i c}{n_{g}} \cdot \frac{-\omega_{0}^{2}}{2 \beta_{0} c^{2}} \sum_{m^{\prime}, n^{\prime}} \int \xi_{-1-m^{\prime},-n^{\prime}} E_{y, m^{\prime}, n^{\prime}} \Theta_{0}^{*}(z) d z, \\
& \frac{\partial R_{y}}{\partial t}=\frac{c}{n_{g}}\left(-i \delta+\frac{g}{2}\right) R_{y}-\frac{c}{n_{g}} \frac{\partial R_{y}}{\partial y}-\gamma R_{y}+\frac{i c}{n_{g}} \cdot \frac{-\omega_{0}^{2}}{2 \beta_{0} c^{2}} \sum_{m^{\prime}, n^{\prime}} \int \xi_{-m^{\prime}, 1-n^{\prime}} E_{x, m^{\prime}, n^{\prime}} \Theta_{0}^{*}(z) d z, \\
& \frac{\partial S_{y}}{\partial t}=\frac{c}{n_{g}}\left(-i \delta+\frac{g}{2}\right) S_{y}+\frac{c}{n_{g}} \frac{\partial S_{y}}{\partial y}-\gamma S_{y}+\frac{i c}{n_{g}} \cdot \frac{-\omega_{0}^{2}}{2 \beta_{0} c^{2}} \sum_{m^{\prime}, n^{\prime}} \int \xi_{-m^{\prime},-1-n^{\prime}} E_{x, m^{\prime}, n^{\prime}} \Theta_{0}^{*}(z) d z .
\end{aligned}
$$

It should be noted that these typographical errors did not alter any numerical results in the paper. 